ARTÍCULO DE INVESTIGACIÓN

\title{
PROPIEDADES PSICOMÉTRICAS DE LA VERSIÓN ARGENTINA DE LA ESCALA REVISADA DE ANSIEDAD Y DEPRESIÓN INFANTIL (RCADS)
}

\author{
PSYCHOMETRIC PROPERTIES OF THE ARGENTINIAN VERSION OF THE REVISED \\ CHILDREN ANXIETY AND DEPRESSION SCALE (RCADS)
}

\section{LEANDRO MARTÍN CASARI ${ }^{1}$, CELESTE DAHER ${ }^{2}$, MARÍA JIMENA ARROYO ${ }^{3}$, DANIELA FERNANDA GONZÁLEZ ${ }^{4}$, VALERIA ESTEFANÍA MORÁN 5 , MIRTA SUSANA ISON ${ }^{6}$ \\ INCIHUSA, CONICET CCT MENDOZA. PONTIFICIA UNIVERSIDAD CATÓLICA ARGENTINA. UNIVERSIDAD DEL ACONCAGUA. UNIVERSIDAD NACIONAL DE CUYO. UNIVERSIDAD SIGLO 21.}

FECHA RECEPCIÓN: 11/12/2020 • FECHA ACEPTACIÓN: 04/05/2021

\begin{abstract}
Para citar este artículo: Casari, L, M., Daher, C., Arroyo, M., González, D., Morán, E., \& Ison M. (2021). Propiedades psicométricas de la versión argentina de la Escala Revisada de Ansiedad y Depresión Infantil (RCADS). Psychologia, 15(1), 13-30. https://doi: 10.21500/19002386.5179
\end{abstract}

\begin{abstract}
Resumen
El principal objetivo de este estudio fue evaluar la validez y confiabilidad de la Escala Revisada de Ansiedad y Depresión Infantil (RCADS). Específicamente, se analizó la validez de contenido, la validez de constructo y la validez de criterio, además de reportar los índices de confiabilidad. La muestra estuvo conformada por 284 escolares, cuyas edades oscilaban entre 8 y 12 años, residentes de la provincia de Mendoza, Argentina. Se emplearon los siguientes instrumentos: Escala Revisada de Ansiedad y Depresión Infantil, el Cuestionario de Depresión Infantil (CDI), y el Cuestionario de Emociones Positivas (CIEP). Para evaluar la validez de contenido se convocaron a 10 jueces expertos, y se encontró un grado de acuerdo aceptable con respecto a la congruencia de los ítems a los factores evaluados. La

1 INCIHUSA, CONICET CCT Mendoza. Facultad de Humanidades y Ciencias Económicas, Pontificia Universidad Católica Argentina. https://orcid. org/0000-0002-0139-0798

2 Facultad de Psicología, Universidad del Aconcagua. Facultad de Humanidades y Ciencias Económicas, Pontificia Universidad Católica Argentina. https:// orcid.org/0000-0002-7838-5911

3 INCIHUSA, CONICET CCT Mendoza. Facultad de Psicología, Universidad del Aconcagua. https://orcid.org/0000-0001-8701-594X

4 Facultad de Psicología, Universidad del Aconcagua. Facultad de Humanidades y Ciencias Económicas, Pontificia Universidad Católica Argentina. https:// orcid.org/0000-0003-1357-4241 5: Universidad Siglo 21. https://orcid.org/0000-0003-3628-163

5 Universidad Siglo 21. ORCID: https://orcid.org/0000-0003-3628-1636

6 INCIHUSA, CONICET CCT Mendoza. Facultad de Psicología, Universidad del Aconcagua. https://orcid.org/0000-0002-3598-982X


validez de constructo fue evaluada comparando modelos alternativos de la RCADS, encontrando índices de ajuste adecuados para un modelo de cinco factores y 25 ítems, así como índices aceptables de confiablidad. Por último, se encontraron correlaciones significativas positivas entre las dimensiones del RCADS con el CDI y correlaciones significativas negativas con los factores del CIEP. Se discuten los resultados en función de los antecedentes, y se señalan limitaciones de la presente investigación.

Palabras claves: Ansiedad, Depresión, Evaluación, Infancia, Psicometría.

\section{Abstract}

The main objective of this study was to evaluate the validity and reliability of the Revised Child Anxiety and Depression Scale (RCADS). Specifically, content validity, construct validity and criterion validity were assessed, in addition to reporting the reliability index. The sample was composed of 284 schoolchildren whose ages ranged from 8 to 12 years. All of them were from the state of Mendoza, Argentina. The following instruments were used: Revised Child Anxiety and Depression Scale, the Child Depression Questionnaire (CDI), and the Positive Emotions Questionnaire (CIEP). To evaluate content validity, 10 expert judges were summoned, and an acceptable degree of agreement was found regarding the congruence of the items to the evaluated factors. The construct validity was evaluated by comparing alternative models of the RCADS, finding adequate fit indices for a model of five factors and 25 items, as well as an acceptable index of reliability. Finally, significant positive correlations were found between the dimensions of the RCADS with the CDI and significant negative correlations with the factors of the CIEP. The results are discussed based on the previous research, and the weakness of this research are pointed out.

Keywords: Anxiety, Depression, Assessment, Childhood, Psychometrics.

\section{Introducción}

La incidencia de problemas de salud mental en la infancia se ha ido acrecentando en las últimas décadas (Gonzalvez et al., 2019), alcanzando entre un $13.4 \%$ y un 20\% de niños, niñas y adolescentes (en adelante NNyA) que poseen algún trastorno mental (Polanczyk et al., 2015; Radwan \& Mallik, 2018; Vasileva et al., 2021). Asimismo, se estima que al menos uno de cada tres NNyA comenzará a padecer el mismo antes de los 16 años (Weisz et al., 2017). Diversos estudios epidemiológicos que se han realizado en distintos países señalan que las patologías más prevalentes son: trastorno depresivo mayor, trastorno por déficit atencional, trastorno oposicionista desafiante, fobia social, trastorno obsesivo compulsivo y trastorno de ansiedad generalizada. Cabe desatacar que todas ellas podrían resumirse en un gran grupo de patologías relacionadas a problemas de ansiedad y depresión infantil (Chen et al., 2019; Deighton et al., 2019: Holbrook et al., 2018; Paul \& Khan, 2019; Radwan \& Mallik, 2018; Yuen et al., 2018). Además, se ha encontrado que en la infancia la comorbilidad entre estas patologías es muy elevada (Holbrook et al., 2018; Vasileva et al., 2021).

La comorbilidad entre ansiedad y depresión infantil ha sido un tema estudiado desde el año 1992, en el cual Brady y Kendall postularon una estrecha relación entre ambas condiciones clínicas. Cummings et al. (2014) proponen tres modelos para intentar explicar esta elevada comorbilidad: 1) Los niños con ansiedad que desarrollan luego una depresión comórbida subsiguiente, esto se daría particularmente desde Fobia Social o Ansiedad por Separación hacia cuadros depresivos; 2) niñas y niñas con una ocurrencia simultánea de ansiedad (particularmente con Ansiedad Generalizada), y depresión; 3) niñas y niñas con trastorno depresivo que luego desarrollan un trastorno de ansiedad, particularmente Fobia Social.

Fruto de la elevada comorbilidad, han surgido modelos alternativos a los categoriales, que intentan explorar la psicopatología desde una perspectiva dimensional. Por ejemplo, Achenbach (1966) propuso un modelo para entender la conducta desadaptativa en la infancia, como un contínuum entre trastornos internalizadores y trastornos externalizadores. Los primeros obedecen 
a trastornos de sobreadaptación, tales como timidez, inhibición, ansiedad de separación, etc.; mientras que los segundos se relacionan con exceso de adaptación, e incluyen conductas relacionadas con la transgresión de normas: agresión, mentira, manipulación, etc. (De la Iglesia, 2017; Genise, 2019).

Por otro lado, a la hora de evaluar la psicopatología infantil surge la necesidad de recurrir a múltiples informantes, dado que se necesita contar con instrumentos que exploren la salud mental desde distintas perspectivas, tales como padres, educadores, pares, además del propio niño o niña. Se ha establecido que padres y maestros pueden ser informantes más precisos sobre trastornos externalizantes durante la infancia, mientras que a través del autorreporte del niño o niña se puede lograr una mejor apreciación de trastornos internalizantes, tales como ansiedad y depresión (Castro-Solano, 2017; Frick et al., 2009). Particularmente en Argentina, existen escasos instrumentos psicométricos que cuentan con adaptaciones locales para evaluar psicopatología o aspectos emocionales en general. Incluso, se debe destacar que hasta el momento no se ha encontrado algún instrumento que incorpore la evaluación de ansiedad y depresión de forma simultánea (Casari \& García, 2019).

Una de las escalas más utilizadas a nivel mundial es la Escala Revisada de Ansiedad y Depresión Infantil (RCADS, por sus siglas en inglés). Se trata de una escala de autoinforme para niños, que evalúa la ansiedad y depresión de forma multidimensional en seis escalas: Trastorno de Ansiedad por Separación, Fobia Social, Trastorno de Ansiedad Generalizada, Trastorno de Pánico, Trastorno Obsesivo Compulsivo y Trastorno Depresivo Mayor (Chorpita et al., 2000). Estos autores no propusieron un nuevo modelo teórico, sino una forma de evaluación que aglutine trastornos de ansiedad y depresión, que sea consistente con la clasificación vigente de aquel momento: la cuarta edición del Manual Diagnóstico y Estadístico de los Trastornos Mentales (American Psychiatric Association [APA], 1994).

Para la validación de la RCADS, se trabajó con una muestra de 1641 NNyA de Hawái, Estados Unidos, con una edad promedio de 12.87 años (DE: 2.82), con un rango de edad de entre 6 y 18 años. La composición por sexo fue: $54.4 \%$ niñas y $45.6 \%$ niños. Como resultado del análisis factorial exploratorio, se extrajeron 6 factores que explicaron el $41.3 \%$ de la varianza. La denominación de estos y su coeficiente de confiabilidad fue: Trastorno de Ansiedad por Separación $(\alpha=.76)$, Fobia Social $(\alpha$ $=.82$ ), Trastorno Obsesivo Compulsivo $(\alpha=.73)$, Trastorno de Pánico $(\alpha=.79)$, Trastorno de Ansiedad Generalizada $(\alpha=.77)$, Trastorno Depresivo Mayor $(\alpha=.76)$. La escala quedó conformada por un total de 47 ítems, con opción de respuesta en escala tipo Likert: Nunca, $A$ veces, Frecuentemente, Siempre (Chorpita et al., 2000).

Luego, se realizó un segundo estudio para evaluar la validez concurrente de la escala con otras medidas de autoreporte. Se trabajó con 246 niños y adolescentes, con una edad promedio 12.20 (DE: 2.89), cuyas edades oscilaron desde 8 hasta 18 años. Como resultado, se encontraron correlaciones entre factores de la RCADS (trastorno depresivo mayor) con el Cuestionario para la Depresión Infantil (CDI, Kovacs, 1980) y otras medidas para evaluar ansiedad (Chorpita et al., 2000). Estos resultados también fueron replicados en investigaciones posteriores que emplearon el CDI en sus estudios de validación de la RCADS (Becker et al., 2019; Chorpita et al., 2005; de Ross et al., 2002; Fontana et al., 2019; Gormez et al., 2016; Muris et al., 2002; Sandín et al, 2010).

Chorpita et al. (2000) señalan el potencial clínico y de investigación de la escala, y recalcan su utilidad por su congruencia con los síndromes de acuerdo con la cuarta edición del Manual Diagnóstico y Estadístico de los Trastornos Mentales (APA, 1994).

También se realizaron estudios comparando población clínica con población escolar encontrando evidencias de validez convergente y discriminante, tanto con entrevistas clínicas como con otras medidas de autoreporte (Chorpita et al., 2005).

Posteriormente, se desarrollaron versiones abreviadas de la escala con menor cantidad de ítems o de factores. La principal ventaja de estas es que reducen el cansancio en los sujetos examinados, ganando en tiempo de administración (Piqueras, Martín-Vivar, et al., 2017).

El primero de estos modelos fue desarrollado por Muris et al. (2002) con NNyA de Holanda. Producto de análisis factorial exploratorio (AFE), se eliminaron ítems que eran inconsistentes con el modelo original de Chorpita et al. (2000), quedando la escala Trastorno Obsesivo Compulsivo conformada por solo dos ítems. Como resultado, se eliminó dicha subescala, y la nueva versión 
25 ítems y cinco factores fue sometida a análisis factorial confirmatorio (AFC), dando buenos índices de ajuste.

El segundo modelo abreviado fue desarrollado por Sandín et al. (2010) para NNyA de España. El interés inicial fue crear una versión más corta, con el fin de reducir el cansancio en los participantes, y se siguieron distintos criterios para su elaboración, entre ellos mantener los factores originales y conversar el mismo número de ítems por subescala. Empleando AFE se retuvieron los reactivos con mayor carga por factor, y, a su vez, se eliminaron aquellos cuyo contenido semántico sea similar. Esta nueva versión fue revisada a través de AFC dando buenos índices de ajuste; quedó conformada por 30 ítems y los seis factores originales.

El tercer modelo abreviado fue publicado por Ebesutani et al. (2012), que incluyó NNyA de Estados Unidos. El mismo se basa en una respuesta al modelo de Muris et al. (2002), donde se priorizó no eliminar la evaluación de indicadores de Trastorno Obsesivo Compulsivo y suprimir ítems redundantes al igual que en el modelo anterior. A su vez, se cuestionaba que la escasa cantidad de ítems puede afectar la confiabilidad de los factores, como en el modelo de Muris et al. (2002). Por ello, usando AFE bifactorial se seleccionaron aquellos ítems que mejor cargaban en un nuevo constructo de Ansiedad Total, y se conversaron, por otro lado, todos los ítems del factor Depresión Mayor. De este modo, el modelo quedó conformado por 25 ítems y dos grandes factores: Ansiedad y Depresión.

Además de la medida de autorreporte, se ha diseñado una versión para padres de la RCADS, con similar estructura en cuanto a factores evaluados, cantidad de ítems y opciones de respuesta. La única diferencia es que son los padres quienes deben completar la misma, con relación a la sintomatología observada en sus hijos (Ebesutani et al., 2010; 2011). Esta escala, además de haber sido utilizada para comparar población clínica y población no clínica de NNyA, también fue empleada para estudiar percepción de sintomatología clínica en sujetos con antecedentes de institucionalización y maltrato infantil (Ebesutani et al., 2015).

Es importante aclarar que la RCADS posee múltiples estudios en diferentes países que avalan su estabilidad transcultural como modelo, al poder ser replicado su estructura con infantes de diferentes culturas. Original- mente se diseñó en Estados Unidos, pero existen adaptaciones en diversos países de Europa (Bouvard et al., 2012; Donnelly et al., 2019; Esbjørn et al., 2012; Mathyssek et al., 2013; Sandín et al. 2010; Kösters, et al., 2015), Asia (Bagheri et al., 2019; Gormez et al., 2016; Mehmood \& Sultan, 2014), Oceanía (de Ross et al., 2002), Centro y Sur de América (Batista \& Martínez, 2013; Fontana et al., 2019; Martínez-González et al., 2020).

Una revisión sistemática reciente señaló que las propiedades psicométricas de la RCADS son satisfactorias en las adaptaciones realizadas en diversos países, indicando que es una de las medidas más utilizadas a nivel mundial para la evaluación de la ansiedad y depresión en niños y adolescentes (Piqueras, Martín-Vivar et al., 2017).

Teniendo en cuenta este recorrido y la escasa disposición de instrumentos de autoinforme que evalúen la afectividad infantil en el medio nacional es que este estudio se propone estudiar las propiedades psicométricas de la RCADS en población escolar de Argentina. Específicamente, se establecieron los siguientes objetivos: analizar la validez de contenido de la RCADS; analizar la validez de constructo de los distintos modelos disponibles de la RCADS y su confiabilidad; analizar la validez convergente de la RCADS con el CDI y la validez divergente con el Cuestionario Infantil de Emociones Positivas (CIEP). Como hipótesis se plantean: 1) los modelos abreviados poseen mejores índices de ajuste que el modelo original; 2) existen correlaciones positivas entre las subescalas de la RCADS con el CDI y correlaciones negativas entre los factores de la RCADS con las dimensiones del CIEP.

\section{Método}

\section{Diseño}

La presente investigación es considerada como un estudio instrumental ya que se propuso el estudio de las propiedades psicométricas de un instrumento de evaluación (Montero \& León, 2007).

\section{Participantes}

La muestra estuvo conformada por 284 escolares de la provincia de Mendoza, Argentina, provenientes de cuatro escuelas: dos de gestión estatal de ámbito urbano marginado, y dos de gestión privada de ámbito urbano, de acuerdo con la clasificación de la Dirección General 
de Escuelas de Mendoza, Argentina [DGE] (Ministerio de Cultura y Educación de la provincia de Mendoza, Argentina, 1985). Las edades oscilaron entre 8 a 12 años, con una media de 10.8 (DE: 1.4). En cuanto al género, 141 escolares pertenecían al género femenino (50.4\%), y 143 eran de género masculino (49.6\%).

\section{Instrumentos}

Escala Revisada de Ansiedad y Depresión Infantil (RCADS) (Chorpita et al., 2000). Es una escala de autoinforme para NNyA que evalúa la ansiedad y depresión de forma multidimensional, en seis escalas: Trastorno de Ansiedad por Separación, Fobia Social, Trastorno de Ansiedad Generalizada, Trastorno de Pánico, Trastorno Obsesivo Compulsivo y Trastorno Depresivo Mayor. La escala está compuesta por 47 ítems y se responde en un formato tipo Likert ( 0 a 3). Cuenta con propiedades psicométricas satisfactorias tanto en análisis factorial exploratorio y confirmatorio, así como niveles aceptables de confiabilidad obtenidos mediante el cálculo del alpha de cronbach y estabilidad test-retest (Chorpita et al., 2000; Chorpita et al., 2005).

Cuestionario de Depresión Infantil (CDI, Kovacs, 1980). Su objetivo es evaluar la severidad del síndrome depresivo en NNyA de 7 a 15 años a través de un único factor. Puede administrarse de manera individual o colectiva, oscilando el tiempo de aplicación entre 10 y 25 minutos, en función de la habilidad lectora del sujeto evaluado. Posee 27 ítems, de tres alternativas cada uno, en los cuales el sujeto informa acerca de síntomas de depresión característicos del rango etario mencionado. Se administró la adaptación argentina de Facio y Batistuta (2001), que cuenta con propiedades psicométricas satisfactorias en validez (a través de análisis factorial exploratorio) y en confiabilidad (valores aceptables de alpha de Cronbach y estabilidad test retest). En la presente muestra los valores de confiabilidad fueron aceptables: $\alpha=.795$

\section{Cuestionario Infantil de Emociones Positivas} (CIEP, Oros, 2014). El Cuestionario Infantil de Emociones Positivas (CIEP) fue diseñado y validado por Oros (2014) con la finalidad de medir la experiencia emocional positiva en niños y niñas de 8 a 12 años. Evalúa cuatro dimensiones: Alegría y Gratitud, Serenidad, Simpatía y Satisfacción Personal. Está compuesto por 23 ítems que se responden en una escala tipo Likert de tres posibles opciones según el grado de acuerdo con la frase que se expresa. La prueba posee propiedades psicométricas aceptables: validez (Análisis Factorial Exploratorio) y confiabilidad (alpha de Cronbach superior a .60 en las cuatro dimensiones). En la presente muestra, se encontraron índices aceptables de confiabilidad en las cuatro dimensiones: Alegría y Gratitud $(\alpha=.750)$, Serenidad $(\alpha=.712)$, Simpatía $(\alpha=.703)$, Satisfacción Personal $(\alpha=.732)$. La escala total arrojó un índice de $\alpha=.791$. A nivel de correlaciones intratest, se encontraron las siguientes correlaciones estadísticamente significativas entre las subescalas, las cuales fueron todas positivas y significativas a nivel .01: Serenidad correlacionó con Alegría y Gratitud $(r=.565)$, Satisfacción Personal $(r=.368)$ y Emocionalidad Positiva Total $(r=.762)$; Alegría y Gratitud correlacionó con Simpatía $(r=.339)$, Satisfacción Personal $(\mathrm{r}=.440)$ y Emocionalidad Positiva Total $(\mathrm{r}=$ .862); Simpatía correlacionó con Emocionalidad Positiva Total $(r=.536)$, y, por último, Satisfacción Personal correlacionó con Emocionalidad Positiva Total ( $\mathrm{r}=.647)$. Todas las correlaciones poseen intensidades cuyos son grandes $(r>.30)$ o muy grandes $(r>.40)$, de acuerdo a los criterios de Funder y Ozer (2019).

\section{Procedimiento}

En primer lugar, se solicitó autorización a los autores originales de la RCADS, para realizar la adaptación local. Además, se preguntó si a pesar de que la RCADS fue creada para ser consistente con los criterios diagnósticos de la cuarta edición del Manual Diagnóstico y Estadístico de Trastornos Mentales (APA, 1994), aun guardaría coherencia con los criterios diagnósticos de la quinta edición de dicho manual (APA, 2013). La respuesta dada por los autores fue que los cambios en los seis trastornos evaluados eran menores, y que existían publicaciones psicométricas acerca de las propiedades psicométricas de la RCADS posteriores a la publicación de la quinta edición (Comunicación Personal, año 2017).

Posteriormente, se solicitó a tres psicólogas bilingües, de diferentes regiones geográficas de Argentina, que realicen una traducción de la versión original de la escala (compuesta por 47 ítems). No se brindaron especificaciones sobre el rango etario o nivel socioeconómico de la población a la cual se aplicaría el instrumento, para que la traducción no fuera sesgada. Luego, se compararon las tres traducciones, y se consultó a las psicólogas 
por el uso de algunos términos. Esta versión preliminar fue retrotraducida del inglés al español (backtranslation), y no se encontraron diferencias importantes. Posteriormente, los autores de la presente investigación analizaron cada ítem para evaluar su posible adecuación a niños y niños de 8 a 12 años.

Seguidamente, se analizó la validez de contenido a través del juicio de expertos. Para ello, se contactó a 10 profesionales expertos en temáticas de infancia relacionadas con evaluación psicológica, psicología clínica e investigación. Se solicitó que evaluaran el grado de congruencia entre el ítem y el constructo mediante una escala Likert de cinco puntos (desde Totalmente en Desacuerdo hasta Totalmente de Acuerdo); también se preguntó acerca de la claridad semántica y sintáctica de cada ítem, y se dejó espacio para observaciones.

Posteriormente, se realizó una muestra piloto con 20 niños y niñas de 8 a 12 años de Mendoza, para analizar la comprensión de los ítems, y hacer modificaciones en la presentación de las consignas.

Por último, se efectuaron leves cambios en la presentación de los ítems, y se conformó la versión final de la escala. La misma fue aplicada a escolares de 8 a 12 años de la provincia de Mendoza.

Antes de la administración, se solicitó el aval del Subcomité Provincial de Ética e Investigación en Salud Mental de la provincia de Mendoza, que fue otorgado previo a la recolección de datos. Se convocaron a niños y niñas de cuatros escuelas de la provincia de Mendoza a través de un consentimiento informado enviado por medio del cuaderno de comunicaciones. Una vez obtenida la firma de este por un adulto responsable, se explicó a cada niño/a en qué consistiría la investigación, y se solicitó su propio asentimiento por escrito. La recolección de datos se realizó en grupos pequeños, en presencia del equipo de investigación a cargo. $\mathrm{Al}$ concluir esta etapa, se realizaron devoluciones individuales en caso de observar indicadores elevados de ansiedad o depresión en los factores de la RCADS. También se confeccionó un informe de devolución grupal a la escuela, y talleres de devolución con las familias.

\section{Análisis de datos}

El análisis de validez de contenido se exploró a través del grado de acuerdo entre los jueces expertos (Es-
cobar-Pérez \& Cuervo-Martínez, 2008). Cada juez debía puntuar en una escala Likert de cinco puntos, el grado de acuerdo con respecto a la pertinencia de cada ítem al constructo evaluado, así como de la claridad semántica y sintáctica. Posteriormente, se analizó el grado de acuerdo a través del cálculo de la $V$ de Aiken, empleando el software Visual Basic. Tomando los criterios de Merino Soto y Livia Segovia (2009), un valor igual o superior a 0.75 , cuyo intervalo de confianza se encuentre comprendido al $99 \%$ es considerado adecuado.

En cuanto a la validez de constructo, los datos se procesaron en primer lugar con el Procesador Estadístico para Ciencias Sociales (SPSS v. 25.0). Para realizar el análisis factorial confirmatorio se empleó el software MPlus 7 y se utilizó el método de estimación de mínimos cuadrados ponderados diagonales (DWLS), adecuado para datos de naturaleza ordinal. Los índices de ajuste considerados fueron el estadístico chi-cuadrado, el Índice de Ajuste Comparativo (CFI), el Índice de TuckerLewis (TLI), considerando para ambos, valores entre .90 y .95 como aceptables a excelentes, el Error Cuadrático Medio de Aproximación (RMSEA) para el que se esperan valores entre .05 y .08, y la ponderada Media Cuadrática Residual (WRMR) esperando valores menores a 1 (Yu \& Muthen, 2002). Para analizar la consistencia interna de la escala se calculó el índice de fiabilidad compuesta $(\omega)$.

Por último, se empleó el coeficiente de correlación de Pearson para explorar la presencia de correlaciones significativas entre la RCADS con el CDI y el CIEP. La intensidad de las correlaciones se clasificó de acuerdo con los criterios de Funder y Ozer (2019), cuyos valores propuestos son: $r=.05$ (efecto muy pequeño), $r=.10$ (efecto pequeño), $r=.20$ (efecto medio), $r=.30$ (efecto grande), $r=.40$ (efecto muy grande).

\section{Resultados}

A continuación, se presentan los resultados detallando el análisis de validez de contenido, validez de constructo y validez de criterio.

\section{Análisis de validez de contenido}

Se contactaron a 10 profesionales que actuaron como jueces expertos por su idoneidad en las temáticas de evaluación psicológica, psicología clínica e investigación sobre niñez. Se les envió una grilla con la versión 
traducida de la RCADS, producto de la depuración y comparación entre las tres traducciones obtenidas por tres psicólogas bilingües, para que se analice la congruencia ítem-constructo, y la adecuación semántica y sintáctica. Se realizó el cálculo de la V de Aiken con el software Visual Basic (Merino Soto \& Livia Segovia, 2009).

Como resultado, los primeros 46 ítems obtuvieron valores aceptables: $V \geq .75$ (IC $99 \%)$. Solo el último ítem, número 47, obtuvo un valor relativamente inferior: $V=.67$ (IC $95 \%$ ), cuyo contenido dice Me siento inquieto $o$ intranquilo. Con relación a la claridad semántica y sintáctica, los valores de los 47 ítems fueron adecuados: $V \geq$ .72 (IC $99 \%$ ). Se hicieron leves modificaciones en la redacción, en función de las sugerencias de los evaluadores.

\section{Análisis de validez de constructo}

Previo a la realización del análisis factorial confirmatorio, se evaluó el patrón de valores perdidos para estimar si respondía a una distribución aleatoria, y de este modo evaluar el porcentaje de estos valores en cada variable en estudio. Se utilizó la rutina de Análisis de Valores Perdidos de SPSS para detectar los valores ausentes. Se observó que estos valores, en ninguna de las variables bajo estudio, superaron el $5 \%$. Aquel ítem con mayor cantidad de casos perdidos $(n=6,2.1 \%)$, fue el número 6, que establece No me divierto tanto como antes, perteneciente al factor Depresión Mayor (Chorpita et al., 2000).
Si consideramos los seis factores del modelo original, la distribución de casos perdidos por dimensiones fue la siguiente: Depresión Mayor $(\mathrm{n}=14)$, Trastorno de Pánico $(n=9)$, Fobia Social $(n=8)$, Ansiedad de Separación $(n=5)$, Ansiedad Generalizada $(n=5)$, Obsesivo Compulsivo $(n=1)$. De este modo, debido al bajo porcentaje de casos perdidos, se conservó la totalidad de la muestra y se procedió a imputar los datos faltantes, siendo cualquier medida de imputación aceptable por el porcentaje bajo de casos perdidos (Hair et al., 2006; Tabachnik \& Fidell, 2007). Se reemplazaron los valores perdidos utilizando el método de imputación Expectation-Maximization (EM).

Por otro lado, se evaluó la presencia de casos atípicos univariados y multivariados. En el primer caso, se tomó el análisis de las puntuaciones tipificadas (z), tomando como criterio \pm 3 . Mientras que los casos atípicos multivariados, fueron examinados con la prueba de distancia de Mahalanobis (Tabachnick \& Fidell, 2007). En ambos casos no se encontraron resultados positivos.

Para el análisis factorial confirmatorio se tomaron en cuenta los cuatros modelos disponibles de la RCADS para NNyA, que fueron mencionados previamente. Los factores e ítems que componen cada modelo se presentan en la Tabla 1.

Tabla 1. Ítems y factores que componen la estructura de cada modelo.

\begin{tabular}{|c|c|c|c|c|}
\hline Escala & $\begin{array}{l}\text { Modelo 1: Chorpita et al. } \\
(2000)\end{array}$ & $\begin{array}{l}\text { Modelo 2: Sandín } \\
(2010)\end{array}$ & $\begin{array}{l}\text { Modelo 3: Murris } \\
(2002)\end{array}$ & $\begin{array}{l}\text { Modelo 4: } \\
\text { Ebesutani et } \\
\text { al. (2012) }\end{array}$ \\
\hline Fobia Social & $\begin{array}{l}4,7,8,12,20,30,32 \\
38,43\end{array}$ & $20,30,32,38,43$ & $4,7,12,30,38$ & \\
\hline Trastorno de pánico & $\begin{array}{l}3,14,24,26,28,34,36 \\
39,41\end{array}$ & $14,26,34,39,41$ & $39,26,28,24,14$ & \\
\hline Depresión Mayor & $\begin{array}{l}2,6,11,15,19,21,25 \\
29,40,47\end{array}$ & $2,6,19,25,29$ & $2,6,15,21,40$ & \\
\hline Ansiedad de Separación & $5,9,17,18,33,45,46$ & $5,9,17,18,46$ & $5,9,17,33,46$ & \\
\hline Ansiedad Generalizada & $1,13,22,27,35,37$ & $1,13,22,27,35$ & $13,22,27,35,37$ & \\
\hline Obsesivo Compulsivo & $10,16,23,31,42,44$ & $10,16,23,31,42$ & & \\
\hline
\end{tabular}


Ansiedad

Depresión
$4,5,13,17$,

$26,27,31,3$

$2,33,36,37$,

$41,42,43$,

44

$2,6,11,15$,

$19,21,25$,

$29,40,47$
Posteriormente, se calcularon los índices de ajuste para cada modelo. Los resultados mostraron que los modelos 1, 2 y 4 presentaron índices de ajuste que, si bien no alcanzaron los valores establecidos, fueron cercanos (Tabla 2). Se revisaron en cada uno de ellos los índices de modificación, encontrando que los efectos y las covariaciones sugeridas daban cuenta de ítems multidimen- sionales, que no se adecuan a la estructura conceptual propuesta, por lo que se decidió no introducir las modificaciones y considerar los índices de ajuste iniciales. Sumado a ello, se encontró que el ítem 47 no presentó pesos de regresión significativos en ninguno de los modelos que lo incluyen (Modelo 1 y 4 ).

Tabla 2. Índices de ajuste de cada modelo

\begin{tabular}{lllllllll}
\hline \hline Modelo & $X^{2}$ & gl & $X^{2} / g l$ & CFI & TLI & RMSEA & WRMR & Dif. $X^{2}$ \\
Modelo 1 & 1336.77 & 974 & 1.37 & .85 & 0.84 & .05 & 1.15 & $970.32 * *$ \\
Modelo 2 & 545.78 & 362 & 1.51 & .86 & .84 & .06 & 1.06 & $170.33 * *$ \\
Modelo 3 & 366.45 & 242 & 1.51 & .90 & .90 & .06 & $\mathbf{0 . 9 8}$ & \\
Modelo 4 & 486.39 & 274 & 1.78 & .85 & .84 & .06 & 1.14 & $119.94 * *$ \\
\hline \hline
\end{tabular}

$$
* * p<.001
$$

En negrita se marcan los índices ajuste aceptables.

${ }^{1}$ Diferencia en valor chi cuadrado con respecto al modelo con mejores índices de ajuste (Modelo 3).

Con respecto al Modelo 3, todos los índices de ajuste fueron satisfactorios, dando cuenta de que es la estructura que mejor se adecua a los datos. Para sustentar esta hipótesis, se realizó un análisis de modelos rivales comparando el Modelo 3 con el resto de los modelos, dando como resultado diferencias significativas en todos los casos.

Los coeficientes de regresión del modelo 3 fueron significativos para todos los ítems $(p \leq .000)$, tomando valores estandarizados entre .31 y .81 . Finalmente, se calculó la fiabilidad compuesta de las escalas, obteniendo .55 para el factor $1, .69$ para el factor 2, .73 para el factor 3, .67 para el factor 4, y .72 para el factor 5 . Se observa que todos los constructos latentes presentaron valores considerados aceptables por la literatura para su uso en investigación, no así para el diagnóstico clínico. Si bien diversos autores postulan valores superiores a .70 como admisibles (George \& Mallery, 2007; Hernández-Sampieri et al., 2014; Hogan, 2004), existe consenso en señalar que el coeficiente de confiabilidad exigido es diferente de acuerdo al propósito de la investigación. Por ejemplo, en la toma de decisiones clínicas se recomiendan coeficientes mayores a .85 (MoralesVallejo, 2007; Pfeiffer, Heslin \& Jones, 1976; Schmitt, 
1996); mientras que, respecto del uso de los test en contextos de investigación, pareciera que es difícil encontrar un valor mínimo para aceptar un coeficiente de fiabilidad como adecuado (Guilford, 1954; Morales-Vallejo, 2007; Nunnally, 1978). También debe agregarse que el valor de confiabilidad depende de la longitud de la escala (cantidad de ítems) (Gliem \& Gliem, 2003; Schimdt, 1996). Entonces, un índice que, si bien resulta menor a lo aceptable en términos generales, puede considerarse útil en situaciones donde el número de ítems es reducido y de este modo, adecuado para tareas de investigación (Loewenthal, 2001). Por ello, es esperable que el modelo original de Chorpita et al. (2000) de 47 ítems, presente mejores índices de confiabilidad.

Seguidamente, se exploró la presencia de correlaciones significativas al interior de la prueba (ver Tabla
3). Como puede observarse, todos los subfactores presentan correlaciones significativas y positivas, de distintas intensidades de acuerdo a los criterios de Funder y Ozer (2019). Aquellas moderadas cuyo valor de correlación supera a $r=.20$ son: Ansiedad de Separación con Ansiedad Generalizada y con Fobia Social. Las correlaciones moderadas que superan el valor de $r=.30$, son: Fobia Social con Trastorno de Pánico y con Depresión Mayor; Depresión Mayor con Ansiedad de Separación. Por último, las correlacionales que superan el valor de $r=.40$ son consideradas muy fuertes, las mismas se dieron entre las siguientes subescalas: Trastorno de Pánico con Depresión Mayor, Ansiedad de Separación y Ansiedad Generalizada, y entre Ansiedad Generalizada con Fobia Social y Depresión Mayor.

Tabla 3. Correlaciones entre factores de la RCADS

\begin{tabular}{|c|c|c|c|c|}
\hline & $\begin{array}{l}\text { Trastorno de } \\
\text { Pánico }\end{array}$ & $\begin{array}{l}\text { Depresión } \\
\text { Mayor }\end{array}$ & $\begin{array}{l}\text { Ansiedad de } \\
\text { Separación }\end{array}$ & Ansiedad Generalizada \\
\hline Fobia Social & $.359 * *$ & $.327 * *$ & $.280 * *$ & $.497 * *$ \\
\hline Trastorno de Pánico & 1 & $.451 * *$ & $.400 * *$ & $.531 * *$ \\
\hline Depresión Mayor & & 1 & $.335 * *$ & $.480 * *$ \\
\hline $\begin{array}{l}\text { Ansiedad de } \\
\text { Separación }\end{array}$ & & & 1 & $.293 * *$ \\
\hline Ansiedad Generalizada & & & & 1 \\
\hline
\end{tabular}

Nota: $* * \mathrm{p}<.01$

\section{Análisis de validez de criterio}

Se exploró por un lado la validez convergente con el Cuestionario de Depresión Infantil (CDI) (Kovacs, 1980), analizando la presencia de correlaciones entre los factores de la RCADS y el valor total del CDI (ver Tabla 4). Como resultado, se encontraron múltiples correla- ciones significativas y positivas, entre los factores de la RCADS y Depresión Infantil, evaluada a través del CDI. Todas las correlaciones poseen un efecto muy fuerte ( $\mathrm{r}$ $>$.40), de acuerdo con los criterios de Funder y Ozer (2019). El único factor de la RCADS que no correlacionó de manera significativa fue Fobia Social. 
Tabla 4. Correlaciones entre factores de la RCADS y CDI

\begin{tabular}{cc}
\hline \hline & CDI_Depresión Infantil \\
\hline RCADS_Fobia Social & .239 \\
RCADS_Trastorno de Pánico & $.647 * *$ \\
RCADS_Ansiedad de Separación & $.547 * *$ \\
RCADS_Ansiedad Generalizada & $.559 * *$ \\
RCADS_Depresión Mayor & $.581 * *$ \\
\hline \hline
\end{tabular}

Nota: $* * p<.01$.

Por último, se evaluó si existían correlaciones entre los factores de la RCADS y un constructo teórico opuesto, como son las Emociones Positivas, para explorar la validez divergente (ver tabla 5). Como puede observarse, se encontraron múltiples correlaciones sigTabla 5. Correlaciones entre factores de RCADS y Emociones Positivas nificativas y negativas, entre los factores de la RCADS y las Emociones Positivas. Las intensidades oscilaron desde pequeñas $(r>.10)$ hasta muy fuertes $(r>.40)$ (Funder $\&$ Ozer, 2019).

\begin{tabular}{|c|c|c|c|c|c|}
\hline & $\begin{array}{l}\text { Alegría } \\
\text { Gratitud }\end{array}$ & Serenidad & Simpatía & $\begin{array}{l}\text { Satisfacción } \\
\text { Personal }\end{array}$ & $\begin{array}{l}\text { Emocionalidad Positiva } \\
\text { total }\end{array}$ \\
\hline Fobia Social_RCADS & $-.241 * *$ & $-.367 * *$ & .075 & $-.171 *$ & $-.265 * *$ \\
\hline Trastorno de Pánico_RCADS & $-.245 * *$ & $-.159 *$ & .103 & -.050 & -.145 \\
\hline Depresión Mayor_RCADS & $-.406 * *$ & $-.369 * *$ & -.054 & $-.268 * *$ & $-.402 * *$ \\
\hline $\begin{array}{l}\text { Ansiedad de Separación__ } \\
\text { RCADS }\end{array}$ & -.147 & $-.236 * *$ & $.181 *$ & -.070 & -.114 \\
\hline $\begin{array}{l}\text { Ansiedad Generalizada_ } \\
\text { RCADS }\end{array}$ &,$- 141 *$ &,$- 229 * *$ &, 106 &,- 132 &,$- 151 *$ \\
\hline
\end{tabular}

Nota. $* \mathrm{p}<.05, * * \mathrm{p}<.01$.

Por último, se consideró el análisis del efecto del sexo y del ámbito de la escuela, según la Dirección General de Escuelas de Mendoza, Argentina (DGE, s.f.). Por lo tanto, las escuelas fueron recategorizadas en urbano marginales $(n=214)$, y urbanas $(n=70)$. Seguidamente, se realizó un Análisis Multivariado de la Varianza (MANOVA) introduciendo como factores fijos el sexo de los participantes y el ámbito de la escuela, en tanto que se ingresaron los cinco factores de la RCADS como variables dependientes. 
Los resultados revelaron un único efecto significativo para la variable gestión de la escuela, $\lambda$ Wilks .901, $F$ $(5,276)=6.070, p \leq .01, \eta^{2}=.099$. Mediante el Análisis de Varianza Univariado (ANOVA), se observó que la diferencia significativa en la gestión de la escuela, se manifestaba en las subescalas de Trastorno de Pánico ( $F$ $\left.(1)=14.03, p<.01, \eta^{2}=.048\right)$, Depresión Mayor $(F$ $\left.(1)=15.94, p<.01, \eta^{2}=.052\right)$ y Ansiedad de Separación $\left(F(1)=17.47, p<.01, \eta^{2}=.059\right)$. En todos los casos, las medias superiores fueron obtenidas por escolares que asisten a escuelas urbano-marginales. También se encontró en la prueba ANOVA un efecto significativo en la variable sexo en la subescala Ansiedad de Separación $\left(F(1)=4.648, p<.05, \eta^{2}=.016\right)$, donde las medias superiores las obtuvieron los participantes del sexo femenino.

\section{Discusión}

El propósito de este artículo fue evaluar las propiedades psicométricas de la RCADS, por lo que se analizó la validez de contenido, de constructo y de criterio en una muestra de 284 escolares de Mendoza, Argentina.

La validez de contenido es un aspecto central en evaluación psicológico, porque implica evaluar si una prueba extranjera cumple con los requisitos de equivalencia lingüística y conceptual en la cultura, donde se pretende utilizar el instrumento. Por ello, es que a pesar de que la RCADS cuenta con múltiples estudios de propiedades psicométricas en diferentes países, se decidió comenzar con este proceso (Castro-Solano, 2017).

Con relación a la validez de contenido, casi la totalidad de los ítems obtuvieron valores aceptables, excepto el número 47 que expresa: Me siento inquieto o intranquilo. A diferencia de los ítems anteriores, los jueces expertos no manifestaron un acuerdo mayoritario sobre su pertenencia al factor Depresión Mayor. De todos modos, se decidió conversar dicho ítem y someter la escala a análisis factorial confirmatorio, respetando la estructura original (Chorpita et al., 2000).

Es importante mencionar que este método para explorar la validez de contenido no fue incluido en el diseño original de la escala. Los autores comentan que, a partir de los ítems preliminares, siete expertos en el área de ansiedad y depresión infantil evaluaron los reactivos, y se redujeron en su cantidad (Chorpita et al., 2000).
Sin embargo, no se comenta que se haya aplicado algún criterio estadístico para explorar la validez de contenido, sino que únicamente se da a entender que se trató de una discusión entre expertos.

En investigaciones previas donde se realizó el estudio de las propiedades psicométricas de la RCADS en diferentes culturas, el interés estuvo centrado en la adecuación semántica, pero no se ha encontrado un estudio que repita un análisis a través del criterio interjueces, como el aquí realizado, o al menos que se informe una técnica del análisis de concordancia entre jueces expertos (como ejemplos, pueden mencionarse los últimos estudios de adaptación psicométrica realizados por Donnelly et al., 2019; Fontana et al., 2019; Stahlschmidt et al., 2019). Por ello es importante el estudio de la equivalencia semántica y conceptual en la adaptación de pruebas psicométricas (Castro-Solano, 2017). Fue así como en este estudio se partió analizando la pertinencia del ítem a cada constructo evaluado.

Inclusive en países de habla hispana, una vez que se contaba con la adaptación española realizada por Sandín et al. (2009), se decidió trabajar con dicha versión y no con una traducción de la escala original en idioma inglés. Como ejemplos, se pueden citar los trabajos de adaptación realizados en Chile, Cuba y Colombia (Acosta \& de Regil, 2019; Batista \& Martínez, 2013; Martínez et al., 2020).

También uno podría cuestionar si la RCADS, al estar basada en las descripciones de la cuarta edición del Manual Diagnóstico y Estadístico de los Trastornos Mentales (APA, 1994), sería compatible con la última actualización (APA, 2013). Sin embargo, tampoco se encontraron estudios previos sobre la RCADS que reflexionen al respecto. En el presente estudio se consultó sobre su pertinencia al autor original de la escala (Comunicación Personal, 2017), donde se dio una respuesta afirmativa respecto a su utilidad posterior a la quinta edición del manual mencionado. Además, para corroborar su adecuación, también se analizó la correspondencia entre los trastornos evaluados a partir de su descripción en ambas ediciones del Manual Diagnóstico y Estadístico de los Trastornos Mentales (APA, 1994; 2013). Asimismo, es importante mencionar que se encuentra un gran número de publicaciones que emplean la RCADS con posterioridad a la publicación del DSM-5, dando cuenta de que no existi- 
rían problemas con su aplicación (Piqueras, Martín-Vivar et al., 2017).

Cabe destacar que, durante este estudio, se prestó especial atención a la validez de contenido a través del análisis del criterio interjueces, considerando la franja etaria para la cual se estaba adaptando la escala en cuestión. En otras investigaciones previas se menciona el rango etario y abarca hasta 10 años de diferencia, al incluir participantes de 8 a 18 años en el mismo estudio de adaptación, sin mencionar si los ítems que evalúan Ansiedad de Separación, por ejemplo, son igualmente válidos para niños y niñas o para adolescentes (Fontana et al., 2019; Martínez-González et al., 2020; Pineda et al., 2018; Stahlschmidt et al., 2019).

Por otro lado, y siguiendo lo realizado en estudios previos, se realizó un análisis de la validez de constructo comparando los distintos modelos empíricos de la RCADS. Como resultado se encontró que el modelo desarrollado por Muris et al. (2002) ofrecía los mejores índices de ajuste. Dicho modelo fue desarrollado a partir de la investigación realizada con 1748 escolares de Holanda (cuya edad oscilaba entre 8 a 15 años), y se compone de cinco factores: Trastorno de Pánico, Ansiedad de Separación, Fobia Social, Ansiedad Generalizada y Depresión Mayor. Como se señaló anteriormente, un meta-análisis reciente demostraba que todas las versiones de la RCADS presentan adecuadas propiedades psicométricas (Piqueras, Martín-Vivar et al., 2017).

Este modelo en particular elimina el Trastorno Obsesivo Compulsivo de la RCADS. El fundamento de su eliminación obedece a criterios psicométricos. Primeramente, Muris et al. (2002) realizaron un análisis factorial exploratorio y observaron que el $25 \%$ de los ítems cargaban en factores diferentes a los originales. Producto de la depuración de estos ítems, el factor Obsesivo Compulsivo solo quedaba representado por dos ítems, por lo que se procedió a su eliminación. Seguidamente, se realizó análisis factorial confirmatorio, observando que la escala resultante formada por cinco factores y 25 ítems, demostrada un buen ajuste psicométrico.

La eliminación de la subescala que evalúa el trastorno Obsesivo Compulsivo también sería coherente con la modificación propuesta en la quinta edición del Manual Diagnóstico y Estadístico de los Trastornos Mentales (APA, 2013), donde dicho trastorno dejó de pertenecer al grupo de trastornos de ansiedad para pasar a constituir un grupo independiente de condiciones clínicas. Por otro lado, Muris et al. (2002) sostenían que era coherente la supresión de dicha escala debido a que las obsesiones son complejas de distinguir en infantes mediante el autorreporte, debido a su escasa capacidad de abstracción a la hora de identificar pensamientos. Otro argumento que puede mencionarse es que, en estudios previos utilizando la RCADS, se encontraron dificultades con la medición de dicho factor en muestras escolares, es decir, que al trabajar con muestras clínicas los resultados eran psicométricamente superiores (Chorpita et al., 2005; Ebesutani et al., 2012).

Como se mencionó anteriormente, la RCADS posee múltiples estudios en diferentes países que avalan su estabilidad transcultural como modelo, al poder ser replicada su estructura con infantes de diferentes culturas. Por dicho motivo, se optó por no emplear Análisis Factorial Exploratorio y aplicar Análisis Factorial Confirmatorio. Como resultado, se encontró que el ajuste del modelo y sus valores de confiabilidad son adecuados en términos psicométricos.

Estudios previos también realizaron una metodología similar donde comparan los índices de ajuste del modelo original propuesto por Chorpita et al. (2000), con otros modelos alternativos, aunque llamativamente, a diferencia del presente estudio donde fueron contrastados con modelos teóricos, es decir, publicados por otros autores, sino que se contrastaron versiones alternativas de la escala original compuestas por dos factores generales o un factor general (Becker et al., 2019; Bouvard et al., 2012; Brown et al., 2013; Chorpita et al., 2005; Donnelly et al., 2019; Fontana et al., 2019; de Ross et al., 2002, Sandín et al., 2009, 2010; Stahlschmidt et al., 2019; Trent et al., 2012). En este estudio, los modelos rivales que se compararon son producto de investigaciones empíricas y propuestos como alternativas al modelo original de Chorpita et al. (2000).

Vale aclarar que la práctica de comparar modelos rivales en una muestra similar puede ser criticada debido a la heterogeneidad de la muestra, en este caso niños y niñas provenientes de distintas escuelas y ámbitos. Sin embargo, en la literatura pueden encontrarse ejemplos de investigaciones que emplean estos análisis estadísticos 
en una única muestra (Casari et al., 2017; Conn et al., 2013; Garrido et al., 2018; Millán et al., 2014).

También se menciona que se encontraron correlaciones significativas y positivas entre todos los factores de la RCADS, dando cuenta de que se trata de escalas relacionadas. Sin embargo, la magnitud de las correlaciones explica que, a pesar de su conexión, también son factores relativamente independientes.

Por último, se exploró la relación de la RCADS con dos pruebas: una para evaluar depresión (Child Depression Inventory) y otra que evalúa un constructo diferente, como son las Emociones Positivas (Oros, 2014). Con respecto a la correlación con Depresión, se encontraron múltiples correlaciones significativas en sentido positivo o directo, no solo con la dimensión Depresión Mayor de la RCADS, sino con las demás subdimensiones. Tanto en el estudio original de validación de la RCADS (Chorpita et al., 2000) como en estudios psicométricos posteriores, se empleó la CDI como uno de los instrumentos para explorar validez convergente, encontrando resultados similares a los aquí reportados (Becker et al., 2019; Chorpita et al., 2005; Fontana et al., 2019; Gormez et al., 2016; Muris et al., 2002; Sandín et al., 2010; de Ross et al., 2002).

También se menciona que las correlaciones entre las subdimensiones de ansiedad de la RCADS con Depresión evaluadas a través del CDI son coherentes con la comorbilidad de ambas condiciones clínicas, y la tendencia actual a considerar ansiedad y depresión como fenómenos subyacentes a diversas patologías mentales, desde una mirada transdiagnóstica (García-Escalera et al., 2016; Martinsen et al., 2019).

También se encontraron correlaciones negativas entre los factores de la RCADS con las dimensiones de Emociones Positivas, aportando evidencias de validez divergente. En estudios previos también se han utilizado instrumentos que evalúan dimensiones positivas, como el afecto positivo y negativo, el autoconcepto, y fortalezas y debilidades, encontrando correlaciones negativas con los factores del RCADS (Muris et al., 2002; Piqueras, Pineda, et al., 2017; Sandín et al., 2010). Se optó por utilizar el Cuestionario de Emociones Positivas, ya que para aportar a la validez ecológica de los instrumentos (Contini, 2018) es deseable trabajar con instrumentos desarrollados en culturas locales, y no únicamente adaptar instrumentos desarrollados en el extranjero.

Por último, se analizó el efecto del género de los participantes, así como de su contexto socioeconómico, evaluado a través de la escuela a la cual asisten. En el primer caso, solo en la escala Ansiedad de Separación se registraron diferencias significativas, encontrando mayores valores en las niñas participantes. Estos resultados también fueron encontrados en estudios previos que emplearon la RCADS como instrumento (Donelly et al., 2019; Fontana et al., 2019; Martínez-González et al., 2020; Muris et al., 2002; Pineda et al., 2018; Sandín et al., 2010). Por otro lado, se detectaron diferencias significativas en tres escalas de la RCADS donde las medias superiores fueron obtenidas por los escolares de ámbitos urbano-marginales. En la literatura, no se encontraron estudios que, empleando la RCADS, hayan realizado este tipo de análisis; sin embargo, existen investigaciones que hablan de la relación entre pobreza y deterioro en la salud mental en niños y niñas (Oros, 2009; Reiss, 2013; Ridley et al., 2020; Yoshikawa et al., 2012).

Para concluir pueden mencionarse algunas limitaciones y perspectivas futuras de investigación. En primer lugar, aquí solo se cuenta con el reporte del propio infante. Si bien se trabajó con una encuesta sociodemográfica para los padres, donde se solicitaba información básica y una breve historia clínica, no se exploró a través de un cuestionario estructurado la presencia de sintomatología clínica. Es sabido que la evaluación psicológica infantil es multi-informante (Castro-Solano, 2017), por lo tanto, en investigaciones futuras se incluirá también la perspectiva de los adultos responsables a través de técnicas como el Child Behavior Check List (Achenbach, 1991).

Otra limitación puede estar asociada a la modalidad de administración: los investigadores recolectaron datos en grupos reducidos de niños, con el propósito de despejar dudas y observar posibles reacciones adversas a partir de la lectura de los ítems. Esto se hizo atendiendo a consideraciones éticas de velar por la integridad emocional de los infantes durante la administración; sin embargo, es desconocido si la presencia de los investigadores pudo haber influido sobre la deseabilidad social.

Por último, el tamaño muestral puede ser pequeño si se compara con otros estudios previos de la RCADS que usaron muestras más amplias, sin embargo, aquí se 
acotó la franja etaria a infantes de 8 a 12 años, mientras que en otras investigaciones se incluyó a escolares de 8 a 18 años (Pineda et al., 2018).

En síntesis, en este artículo se han explorado diferentes aspectos de la validez y confiabilidad de una escala mundialmente utilizada, como es la RCADS, dando cuenta que se trata de un instrumento que permite una exploración de síntomas de ansiedad y depresión comúnmente frecuentes en población infantil. En estudios futuros se avanzará hacia la estandarización del instrumento, ofreciendo valores normativos, y en explorar la validez discriminante contrastando los puntajes de muestras escolares con muestras clínicas para establecer puntos de corte con fines diagnósticos. Por el momento, en contextos clínicos podría aplicarse para evaluar la evolución de sintomatología ansiosa y depresiva, aplicando el instrumento en repetidas oportunidades, ya que por breve extensión puede ser simple de responder para el propio niño o niña.

Finalmente, se destaca la importancia de contar con instrumentos válidos y confiables para la detección temprana de trastornos mentales en niños y niñas de Argentina. No solo porque la niñez constituye un periodo sensible del desarrollo socioafectivo, sino también por el desafío actual que conlleva el aumento significativo en los niveles de ansiedad y depresión infantil como consecuencia de la pandemia por COVID-19 (Erskine et al., 2017; Loades et al., 2020; Sánchez Boris, 2021).

\section{Referencias}

Achenbach, T. M. (1966). The classification of chidren's psychiatric symptoms: A factor-analytic study. $P_{S y-}$ chological Monographs, General and Applied, 80(7), 1-37. https://psycnet.apa.org/doi/10.1037/ h0093906

Achenbach, T. M. (1991). Manual for the Child Behavior Checklist/ 4-18 and 1991 Profile. Burlington: Department of Psychiatry. University of Vermont.

American Psychiatric Association (1994). Diagnostic and Statistical Manual of Mental Disorders ( $4^{\text {th }}$ Ed.). Author

American Psychiatric Association (2013). DSM-5. Diagnostic and statistical manual of mental disorders ( $5^{\text {th }}$ Edition). Author.
Bagheri, Z., Akbari, M., Jafari, P., \& Huedo-medina, T. B. (2019). Assessing the measurement invariance of the RCADS-25 questionnaire across gender and child-parent dyads in the presence of multilevel data. Quality of Life Research, 28(3), 829-840. https://doi.org/10.1007/s11136-018-2049-3

Batista, G. S., \& Martínez, Y. A. S. (2013). La RCADS-30: una técnica psicológica para evaluar conjuntamente la ansiedad y depresión en niños y adolescentes. Valoración de las propiedades psicométricas. Cuadernos de educación y desarrollo, 38, 1-12.

Bouvard, M., Dacquin, F., \& Denis, A. (2012). Étude de la validité de l'échelle d'anxiété et de dépression révisée (RCADS) et de la grille d'évaluation des troubles anxieux forme révisée (SCARED-R). Journal de thérapie comportementale et cognitive, 22(4), 175181. https://doi.org/10.1016/j.jtcc.2012.09.003

Brady, E. U., \& Kendall, P. C. (1992). Comorbidity of anxiety and depression in children and adolescents. Psychological Bulletin, 111 (2), 244-255.

Casari, L. M., \& García, C. (2019). La evaluación psicológica en la clínica de niños y adolescentes. En G. Genise, N. Genise, y L. Crocamo (Comps.), Manual de Psicoterapia y Psicopatología de niños y adolescentes (pp.63-78). Akadia.

Casari, L., Morán, V., \& Ison, M. (2017). Cuestionario de estilo personal del terapeuta: análisis factorial confirmatorio de modelos rivales con psicoterapeutas argentinos. Psychologia, 11 (2), 69-84. https://doi. org/10.21500/19002386.2725

Castro-Solano, A. (2017). La evaluación psicológica infantil. Aspectos conceptuales. En A. Castro-Solano \& M. Fernández Liporace (Eds.), La evaluación psicológica en niños. Técnicas de screening y diagnóstico (pp. 23-40). Paidós.

Chen Y-L, Chen WJ, Lin K-C, Shen L-J, Gau SS-F (2019). Prevalence of DSM- 5 mental disorders in a nationally representative sample of children in Taiwan: methodology and main findings. Epidemiology and Psychiatric Sciences, 29. https://doi. org/10.1017/S2045796018000793

Chorpita, B. F., Moffitt, C. E., \& Gray, J. (2005). Psychometric properties of the Revised Child Anxiety and Depression Scale in a clinical sample. Beha- 
viour research and therapy, 43(3), 309-322. https:// doi.org/10.1016/j.brat.2004.02.004

Chorpita, B.F., Yim, L., Moffi tt, C.E., Umemoto, L.A., \& Francis, S.E. (2000). Assessment of symptoms of DSMIV anxiety and depression in children: A Revised Child Anxiety and Depression Scale. Behaviour Research and Therapy, 38 (8), 835-855. https:// doi.org/10.1016/S0005-7967(99)00130-8

Conn, H., Medrano, L. A., \& Moretti, L. (2013). Adaptación del cuestionario de alianza de ayuda revisado versión paciente (HAQ-II-P) para la población de cordobeses. Terapia psicológica, 31(2), 165-174. http://dx.doi.org/10.4067/S071848082013000200003

Cummings, C. M., Caporino, N. E., \& Kendall, P. C. (2014). Comorbidity of anxiety and depression in children and adolescents: 20 years after. $P_{\text {sycho- }}$ logical Bulletin, 140(3), 816-845. http://dx.doi. org/10.1037/a0034733.

De la Iglesia, G. (2017). Instrumentos para la evaluación psicológica en niños. En En A. Castro Solano \& M. Fernández Liporace (Eds.), La evaluación psicológica en niños. Técnicas de screening y diagnóstico (pp. 5780). Paidós.

De Ross, R., Gullone, E., \& Chorpita, B. (2002). The Revised Child Anxiety and Depression Scale: A Psychometric Investigation with Australian Youth. Behaviour Change, 19(2), 90-101. https:// doi.org/10.1375/bech.19.2.90

Deighton, J., Lereya, S. T., Casey, P., Patalay, P., Humphrey, N., \& Wolpert, M. (2019). Prevalence of mental health problems in schools: poverty and other risk factors among 28000 adolescents in England. The British Journal of Psychiatry, 215(3), 565-567. https://doi.org/10.1192/bjp.2019.19

Donnelly, A., Fitzgerald, A., Shevlin, M., \& Dooley, B. (2019). Investigating the psychometric properties of the revised child anxiety and depression scale (RCADS) in a non-clinical sample of Irish adolescents. Journal of Mental Health, 28(4), 345-356. https://doi.org/10.1080/09638237.2018.1437604 Ebesutani, C., Bernstein, A., Nakamura, B. J., Chorpita, B. F., \& Weisz, J. R. (2010). A psychometric analysis of the Revised Child Anxiety and Depression Scale-Parent Version in a clinical sample. Journal of abnormal child psychology, 38(2), 249-260. https:// doi.org/10.1007/s10802-009-9363-8

Ebesutani, C., Chorpita, B.F., Higa-McMillan, C.K., Nakamura, B.J., Regan, J., \& Lynch, R.E., (2011). A psychometric analysis of the Revised Child Anxiety and Depression Scales - Parent Version in a school sample. Journal of abnormal child psychology, 39, 173-185. https://doi.org/10.1007/s10802010-9460-8

Ebesutani, C., Reise, S. P., Chorpita, B. F., Ale, C., Regan, J., Young, J., Higa-McMillan, C., \& Weisz, J. R. (2012). The Revised Child Anxiety and Depression Scale-Short Version: Scale reduction via exploratory bifactor modeling of the broad anxiety factor. Psychological assessment, 24(4), 833845. https://doi.org/10.1037/a0027283

Ebesutani, C., Tottenham, N., \& Chorpita, B. (2015). The revised child anxiety and depression scaleparent version: Extended applicability and validity for use with younger youth and children with histories of early-life caregiver neglect. Journal of Psychopathology and Behavioral Assessment, 37(4), 705-718. https://doi.org/10.1007/s10862-0159494-x

Erskine, H. E., Baxter, A. J., Patton, G., Moffitt, T. E., Patel, V., Whiteford, H. A., \& Scott, J. G. (2017). The global coverage of prevalence data for mental disorders in children and adolescents. Epidemiology and psychiatric sciences, 26(4), 395-402. https://doi. rog/10.1017/S2045796015001158

Esbjørn, B. H., Sømhovd, M. J., Turnstedt, C., \& Reinholdt-Dunne, M. L. (2012). Assessing the Revised Child Anxiety and Depression Scale (RCADS) in a national sample of Danish youth aged 8-16 years. PLoS One, 7(5), e37339. https://doi. org/10.1371/journal.pone.0037339

Escobar-Pérez, J., \& Cuervo-Martínez, Á. (2008). Validez de contenido y juicio de expertos: una aproximación a su utilización. Avances en medición, 6(1), 27-36.

Facio, A., \& Batistuta, M. (2001). What makes Argentinian girls unhappy? A cross-cultural contribution to understanding gender differences in depressed mood during adolescence. Journal of adolescen- 
ce, 24(5), 671-680. https://doi.org/10.1006/ jado.2001.0420

Fontana, F. E., Da Silva, M. P., Mazzardo, O., Choi, S. I., Lumi, P., De Campos, W., \& Chorpita, B. (2019). Cross-Cultural Adaptation and Psychometric Assessment of the Revised Child Anxiety and Depression Scale in Brazilian Youth. Journal of Asia Pacific Counseling, 9(2), 1-16. https://doi. org/10.18401/2019.9.2.1

Funder, D. C., \& Ozer, D. J. (2019). Evaluating effect size in psychological research: Sense and nonsense. Advances in Methods and Practices in Psychological Science, 2(2), 156-158. https://doi. org/10.1177/2515245919847202

García-Escalera, J., Chorot, P., Valiente, R. M., Reales, J. M., \& Sandín, B. (2016). Efficacy of transdiagnostic cognitive-behavioral therapy for anxiety and depression in adults, children and adolescents: A meta-analysis. Revista de Psicopatologia y Psicologia Clinica, 2 l(3), 147-175. https://doi.org/10.5944/ rppc.vol.21.num.3.2016.17811

Garrido, S. J., Moran, V. E., Azpilicueta, A. E., Cortez, F. D., Arbach, K., \& Cupani, M. (2018). Análisis de modelos rivales unidimensionales y bidimensionales de la escala breve de autocontrol en estudiantes universitarios argentinos. Psicodebate, 18 (2), 2637. http://dx.doi.org/10.18682/pd.v18i2.745

Genise, N. (2019). Introducción a la psicopatología dimensional. En G. Genise, N. Genise y L. Crocamo (Comps.), Manual de Psicopatología y Psicoterapia de niños y adolescentes (pp. 79-102). Akadia.

George, D., \& Mallery, P. (2007). SPSS for Windows: Step by step 14.0 update (7 Ed.). Allyn \& Bacon.

Gliem, J. A., \& Gliem R. R. (2003). Calculating, Interpreting, and Reporting Cronbach's Alpha Reliability Coefficient for Likert-Type Scales. Paper presentado en Midwest Research-to-Practice Conference in Adult, Continuing, and Community Education. The Ohio State University, Columbus, Estados Unidos.

Gonzalvez, M. T., Espada, J. P., \& Orgilés, M. (2019). El estado actual de la psicoterapia en niños y adolescentes. En G. Genise, N. Genise, y L. Crocamo (Comps.), Manual de Psicoterapia y Psicopatología de niños y adolescentes (pp. 1-16). Akadia.
Gormez, V., Kilincaslan, A., Orengul, A. C., Ebesutani, C., Kaya, I., Ceri, V., Filiz, M. y Chorpita, B. (2016). Psychometric properties of the Turkish translation of the revised child anxiety and depression scale-child version (RCADS-CV) in a clinical sample. Psychiatry and Clinical Psychopharmacology, 27(1), 84-92. https://doi.org/10.1080/24750573 .2017 .1297494 .

Guilford, J. P. (1954). Psychometric methods (2 Ed.). McGraw-Hill.

Hair, J. F., Black, W. C., Babin, B. J., Anderson, R. E., \& Tatham, R. L. (2006). Multivariate data analysis (6 Edition). Pearson Education.

Hernández-Sampieri, R.; Fernández-Collado \& BaptistaLucio, C. B. (2014). Metodología de la investigación (6 Ed.). Mc Graw-Hill Interamericana Editores.

Hogan, T. (2004). Pruebas psicológicas. Una introducción práctica. El Manual Moderno.

Holbrook, J., Bitsko, R. H., Danielson, M., Cree, R., Swartz, N., Almeida, A., \& Cuffe, S. P. (octubre, 2018). Prevalence and Co-Occurrence of ParentReported Mental, Behavioral, and Developmental Disorder Diagnoses in US Children: National Survey of Children's Health, 2016. En M. Moretti (Presidencia). Adolescence, Trauma, and Attachment: Translating Research into Practice. Simposio llevado a cabo en AACAP's $65^{\text {th }}$ annual meeting of Washington State Convention Center, Seattle, WA, EEUU.

Kösters, M. P., Chinapaw, M. J., Zwaanswijk, M., van der Wal, M. F., \& Koot, H. M. (2015). Structure, reliability, and validity of the revised child anxiety and depression scale (RCADS) in a multi-ethnic urban sample of Dutch children. BMC psychiatry, 15(1), 132. https://doi.org/10.1186/s12888-015-0509-7

Kovacs, M. (1980). Rating scales to assess depression in preschool children. Acta Paedopsychiatry, 46, 305 315.

Loades, M. E., Chatburn, E., Higson-Sweeney, N., Reynolds, S., Shafran, R., Brigden, A., Linney, C., McManus, M. N., Borwick, C., \& Crawley, E. (2020). Rapid Systematic Review: The Impact of Social Isolation and Loneliness on the Mental Health of Children and Adolescents in the Context of COVID-19. Journal of the American Academy of Child and 
Adolescent Psychiatry, 59(11), 1218-1239. https:// doi.org/10.1016/j.jaac.2020.05.009

Loewenthal, K. M. (2001). An introduction to psychological tests and scales. Psychology Press.

Martínez-González, A. E., Veas, A., \& Piqueras, J. A. (2020). 30-item version of the revised child anxiety and depression scale in Chilean adolescents: psychometric properties. Current Psychology, 1-11. https://doi.org/10.1007/s12144-020-00934-7

Martinsen, K. D., Rasmussen, L. M. P., Wentzel-Larsen, T., Holen, S., Sund, A. M., Løvaas, M. E. S., Patras, J., Kendall, P. C., Waaktaar, T., \& Neumer, S.-P. (2019). Prevention of anxiety and depression in school children: Effectiveness of the transdiagnostic EMOTION program. Journal of Consulting and Clinical Psychology, 87(2), 212-219. https://doi. org/10.1037/ccp0000360

Mathyssek, C. M., Olino, T. M., Hartman, C. A., Ormel, J., Verhulst, F. C., \& Van Oort, F. V. (2013). Does the Revised Child Anxiety and Depression Scale (RCADS) measure anxiety symptoms consistently across adolescence? The TRAILS study. International journal of methods in psychiatric research, 22(1), 27-35. https://doi.org/10.1002/mpr.1380

Mehmood, T., \& Sultan, S. (2014). Translation and adaptation of revised children's anxiety and depression scale. International Journal of Liberal Arts and Social Science, 2(5), 95-106.

Merino-Soto, C. M., \& Livia-Segovia, J. L. (2009). Intervalos de confianza asimétricos para el índice la validez de contenido: Un programa Visual Basic para la V de Aiken. Anales de Psicología/Annals of Psychology, 25(1), 169-171.

Millán, A. C., García-Álvarez, D. J., y D’Aubeterre López, M. E. (2014). Efecto de la inteligencia emocional y flujo de trabajo sobre estresores y bienestar psicológico: análisis de ruta en docentes. Revista Colombiana de Psicología, 23(1), 207-228. https://doi. org/10.15446/rcp.v23n1.37676

Ministerio de Cultura y Educación de la Provincia de Mendoza. (27, febrero 1985). Estatuto docente [313]. https://bases.mendoza.edu.ar/Inicial/pdf/ suplencias/313.pdf
Morales-Vallejo, P. (2007). Estadística aplicada a las ciencias sociales. La fiabilidad de los tests y escalas. Universidad Pontificia Comillas.

Muris, P., Meesters, C., \& Schouten, E. (2002). A brief questionnaire of DSM-IV-defined anxiety and depression symptoms among children. Clinical Psychology \& Psychotherapy: An International Journal of Theory \& Practice, 9(6), 430-442. https://doi. org/10.1002/cpp.347

Nunnally, J.C. (1978). Psychometric Theory. McGraw-Hill.

Oros, L. (2009). El valor adaptativo de las emociones positivas. Una mirada al funcionamiento psicológico de los niños pobres. Interamerican Journal of Psychology, 43(2), 288-296.

Oros, L.B. (2014). Nuevo cuestionario de emociones positivas para niños. Anales de Psicología, 30(2), 522-529. https://dx.doi.org/10.6018/analesps.

Paul, M. A., \& Khan, W. (2019). Prevalence of childhood mental disorders among school children of Kashmir Valley. Community mental health journal, 55(6), 1031-1037. https://doi.org/10.1007/s10597018-0253-9

Pfeiffer, J. W., Heslin, R., \& Jones, J. E. (1976). Instrumentation in human relations training: A guide to 92 instruments with wide application to the behavioral sciences. University Associates.

Pineda, D., Martín-Vivar, M., Sandín, B., \& Piqueras, J. A. (2018). Factorial invariance and norms of the 30-item shortened-version of the revised child anxiety and depression scale (RCADS-30). Psicothema, 30, 232-237. https://doi.org/10.7334/ psicothema2017.276.

Piqueras, J. A., Martín-Vivar, M., Sandin, B., San Luis, C., \& Pineda-Sánchez, D. (2017). The Revised Child Anxiety and Depression Scale: A systematic review and reliability generalization meta-analysis. Journal of Affective Disorders, 218, 153-169. https://doi. org/10.1016/j.jad.2017.04.022

Piqueras, J. A., Pineda, D., Martin-Vivar, M., \& Sandín, B. (2017). Confirmatory factor analysis and psychometric properties of the Revised Child Anxiety and Depression Scale-30 (RCADS-30) in clinical and non-clinical samples. Revista de Psicopatología y Psicología Clínica, 22(3), 183-196. https://doi. org/10.5944/rppc.vol.22.num.3.2017.19332 
Polanczyk, G. V., Salum, G. A., Sugaya, L. S., Caye, A., \& Rohde, L. A. (2015). Annual research review: A meta-analysis of the worldwide prevalence of mental disorders in children and adolescents. Journal of child psychology and psychiatry, 56(3), 345-365. https://doi.org/10.1111/jcpp.12381

Radwan, R. B., \& Mallik, C. I. (2018). 2.7 Psychiatric Disorders Among 14-to 17-Year-Old School-Going Bangladeshi Adolescents. Journal of the American Academy of Child \& Adolescent Psychiatry, 57(10), S161.

Reiss, F. (2013). Socioeconomic inequalities and mental health problems in children and adolescents: a systematic review. Social science \& medicine, 90, 24-31. http://dx.doi.org/10.1016/j.socscimed.2013.04.026

Ridley, M., Rao, G., Schilbach, F., \& Patel, V. (2020). Poverty, depression, and anxiety: Causal evidence and mechanisms. Science, 370, 1-12. https://doi. org/10.1126/science.aay0214

Sánchez Boris, I. M. (2021). Impacto psicológico de la COVID-19 en niños y adolescentes. Medisan, 25(1), 123-141.

Sandín, B., Chorot, P., Valiente, R. M., \& Chorpita, B. F. (2010). Development of a 30-item version of the Revised Child Anxiety and Depression Scale. Revista de Psicopatología y Psicología Clínica, 15(3), 165-178. https://doi.org/10.5944/rppc.vol.15. num.3.2010.4095

Schmitt, N. (1996). Uses and Abuses of Coefficient Alpha. Psychological Assesment, 8(4), 350-353. http:// dx.doi.org/10.1037/1040-3590.8.4.350

Tabachnick, B. L., \& Fidell, L. S. (2007). Using multivariate statistics. (5thEd.). Pearson Education, Inc.

Vasileva, M., Graf, R. K., Reinelt, T., Petermann, U., \& Petermann, F. (2021). Research review: A metaanalysis of the international prevalence and comorbidity of mental disorders in children between 1 and 7 years. Journal of Child Psychology and Psychiatry, 62(4), 372-381. https://doi.org/10.1111/ jсpp. 13261

Weisz, J., Kuppens, S., Ng, M., Eckshtain, D., Ugueto, A., Vaughn-Coaxum, R., Chu, B. (2017). What five decades of research tells us about the effects of youth psychological therapy: a multilevel meta- analysis and implications for science and practice. American Psychologist, 72(2), 79-117. https:// dx.doi.org/10.1037/a0040360

Yoshikawa, H., Aber, J. L., \& Beardslee, W. R. (2012). The effects of poverty on the mental, emotional, and behavioral health of children and youth: implications for prevention. American Psychologist, 67(4), 272-284. https://doi.org/10.1037/a0028015

Yu, C. Y., \& Muthén, B. (2002). Evaluation of model fit indices for latent variable models with categorical and continuous outcomes. Paper presented at the annual meeting of the American Educational Research Association, New Orleans, LA.

Yuen, W. W., Liu, L. L., \& Tse, S. (2018). Adolescent mental health problems in Hong Kong: a critical review on prevalence, psychosocial correlates, and prevention. Journal of Adolescent Health, 64(6), S73-S85. https://doi.org/10.1016/j.jadohealth.2018.10.005 